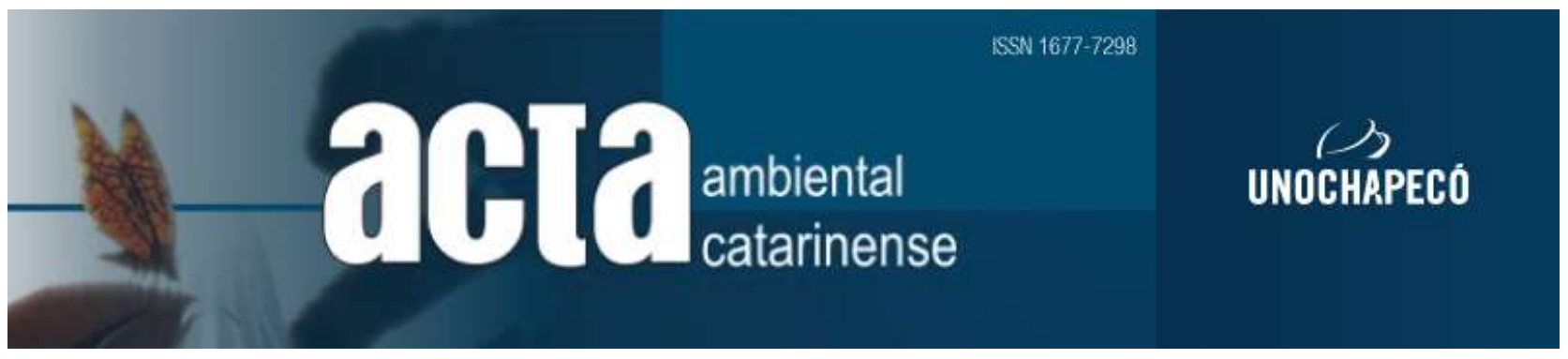

\title{
TOXICIDADE DE ÓLEOS MINERAIS E VEGETAIS NO MANEJO DE PLANOCOCCUS CITRI
}

\author{
Lorena Contarini Machado ${ }^{1 *}$ \\ Hugo José Gonçalves dos Santos Junior ${ }^{2}$ \\ Flávio Neves Celestino ${ }^{3}$ \\ Laura Vaillant Ribeiro Mauri ${ }^{4}$ \\ Ingrid Schimidt Kaiser ${ }^{5}$
}

\begin{abstract}
Resumo
A cochonilha-da-roseta, Planococcus citri, destaca-se no grupo de pragas do cafeeiro, podendo ocasionar perdas à produção. Em virtude da escassez de produtos recomendados para o manejo dessa praga objetivou-se avaliar a atividade inseticida de diferentes óleos vegetais e minerais a $P$. citri. Assim, avaliou-se em laboratório os óleos vegetais de soja, canola, girassol, milho, mamona e nim e os minerais Assist ${ }^{\circledR}$ e Naturol ${ }^{\circledR}$ aplicando-os na concentração de 3\% sobre arenas contendo disco foliar de café com dez ninfas de segundo ínstar. Para os óleos com mortalidade acima de $90 \%$ foi estimada a $\mathrm{CL}_{50}$. Todos os óleos minerais testados apresentaram toxicidade à $P$. citri com a mortalidade variando entre 46,66 a $100 \%$. A CL50 obtida com óleos de nim, Assist ${ }^{\circledR}$ e Naturol ${ }^{\circledR}$ foram semelhantes, sendo respectivamente, 0,$67 ; 0,89$ e 0,76\%. Portanto, após avaliar a fitotoxicidade ao cafeeiro em testes de campo e condições adequadas de pulverização, os óleos vegetais e minerais avaliados podem ser opções viáveis ao manejo integrado da Cochonilha-daroseta.
\end{abstract}

Palavras-chave: Inseticidas botânicos, cochonilha-da-roseta, cafeicultura.

\begin{abstract}
\footnotetext{
1 Doutoranda do Programa de Pós-Graduação em Produção Vegetal pela Universidade Federal do Espírito Santo.

2 Docente no Programa de Pós-Graduação em Produção Vegetal pela Universidade Federal do Espírito Santo.

3 Docente na Pós-graduação Lato Sensu em Cafeicultura Sustentável pelo Instituto Federal de Educação, Ciência e Tecnologia do Sudeste de Minas Gerais.

4 Doutoranda do Programa de Pós-Graduação em Produção Vegetal pela Universidade Federal do Espírito Santo.

5 Doutoranda do Programa de Entomologia pela Universidade de Sã Paulo.

*E-mail: lorenarini@hotmail.com
}

Cochineal-of-rosette, Planococcus citri, stands out in the group of pests of coffee, which may cause losses to production. Due to the scarcity of products recommended for the management of this pest aimed to evaluate the insecticidal activity of vegetable and mineral oils to P. citri. Thus, we 
evaluated laboratory vegetable oils soybean, canola, sunflower, corn, castor bean and nim and minerals Assist ${ }^{\circledR}$ and ${ }^{\circledR}$ Naturol applying them to the concentration of $3 \%$ on leaf disc arenas containing coffee with ten nymphs second instar. For oils with mortality above $90 \%$ was estimated $\mathrm{LC}_{50}$. All mineral oils tested were toxic to P. citri with mortality ranging from 46.66 to $100 \%$. The $\mathrm{LC}_{50}$ obtained with oils of nim, Assist ${ }^{\circledR}$ and Naturol ${ }^{\circledR}$ were similar, being respectively $0.67,0.89$ and $0.76 \%$. Therefore, after evaluating phytotoxicity in the coffee field and ideally spray tests, vegetable oils and minerals reviews may be viable options for integrated management of Cochineal-of-rosette.

Keywords: Botanical insecticides, cochineal-of-rosette, coffee crop.

\section{INTRODUÇÃO}

O Brasil destaca-se como o maior produtor mundial de café, sendo o principal produtor de Coffea arabica L. (Rubiaceae) o segundo maior produtor de Coffea canephora Pierri \& Froenher (Rubiaceae) (NISHIJIMA et al., 2012). A área nacional cafeeira (arábica e conilon) totaliza 2,16 milhões de hectares, e desses 404,3 mil hectares são representados apenas por conilon, cujo no Espírito Santo está a maior área, aproximadamente 265,2 mil hectares, demonstrando a relevância socioeconômica da cafeicultura, principalmente na agricultura familiar (CONAB, 2020).

Contudo, essa atividade agrícola enfrenta problemas que interferem na produção, dentre os quais os fitossanitários, como exemplo: a incidência de insetos-praga. Diante desse contexto, a espécie Planococcus citri Risso (Pseudococcidae) conhecida como Cochonilha-da-roseta, destaca-se no grupo de insetos-praga do cafeeiro, uma vez que pode provocar danos superiores a $30 \%$ (SANTACECÍLIA et al., 2002; FORNAZIER et al., 2009; PICANÇO et al., 2015). Apesar disso, não há nenhum inseticida para essa praga na cultura do café (MAPA, 2020).

Tendo em vista a ausência de produtos recomendados, medidas inadequadas de manejo são adotadas, impulsionando o uso de inseticidas químicos sintéticos impróprios como uma forma de controle imediato da praga. Entretanto, as consequências do uso indiscriminado destes produtos podem ocasionar outros problemas, tais como, interferir em organismo não alvo, selecionar organismos resistentes e expor o ambiente e a saúde humana a riscos de contaminação (PIMENTEL et al., 2006; ISMAN, 2006; TIILIKKALA et al., 2011; ZIBAEE, 2011; MRUTHUNJAYASWAMY et al., 2019).

Portanto, a busca por medidas de manejo é importante para minimizar os impactos ocasionados pelo uso intensivo e inadequado de inseticidas químicos sintéticos (ISMAN, 2006; MRUTHUNJAYASWAMY et al., 2019). Assim, no agroecossistema que adota as boas práticas agrícolas, tais como o uso de produtos seletivos aos inimigos naturais, permite a manutenção dos agentes de controle biológico natural, os quais auxiliam no controle populacional dos insetos-praga (TIILIKKALA et al., 2011; ZIBAEE, 2011).

Considerando outros métodos ao uso dos inseticidas sintéticos, pode-se destacar os inseticidas botânicos, os quais podem ser considerados seletivos aos inimigos naturais, isso dependendo das concentrações e formas de aplicação (ISMAN, 2006; SILVA et al., 2007; LOPES et al., 2009; ZORZETTI et al., 2012).

Dentre os inseticidas botânicos, o óleo de nim, extraído da semente de Azadirachta indica A. Juss. (Meliaceae) é um dos mais utilizados por apresentar atividade inseticida comprovada para muitas espécies de insetospraga (ISMAN, 2006; KUMAR et al., 2019; FITE et al., 2020). Outra espécie vegetal que apresenta potencial inseticida é a mamona, Ricinus communis L. (Euphorbiaceae), tanto na forma de extratos vegetais como também o 
próprio óleo obtido das sementes (RAMOSLÓPEZ et al., 2010; RONDELLI et al., 2011; BESTETE et al., 2011; CELESTINO et al., 2015; KUMAR et al., 2019). No entanto, ainda são escassos na literatura estudos direcionados ao manejo de P. citri. Assim, objetivou-se avaliar a atividade inseticida de diferentes óleos vegetais e minerais a $P$. citri.

\section{Material e Métodos}

Os experimentos foram desenvolvidos no Laboratório de Entomologia do Núcleo de Desenvolvimento Científico e Tecnológico em Manejo Fitossanitário de Pragas (NUDEMAFI), Universidade Federal do Espírito Santo (UFES), em Alegre-ES. Esses foram conduzidos em câmara climatizada a 25 $\pm 1^{\circ} \mathrm{C}$, umidade relativa (UR) de $70 \pm 10 \%$ e fotofase de $12 \mathrm{~h}$.

\subsection{Multiplicação e manutenção de P. citri}

Os insetos foram provenientes de lavoura de café-conilon localizada na área experimental do Centro de Ciências Agrárias da Universidade Federal do Espírito Santo. Posteriormente foram transferidos para abóbora-japonesa que serviram de substrato alimentar, constituindo após a multiplicação a "colônia mãe". Assim, novas abóboras foram colocadas próximas a "colônia mãe" visando o aumento da população em laboratório. Após a fase de transferência de ninfas para abóboras novas, os frutos foram transferidos $\mathrm{e}$ acondicionados em estantes sobre caixa de acrílico (gerbox) para que ocorresse o desenvolvimento sobre a superfície do substrato, conforme adaptação da metodologia empregada por Sanches e Carvalho (2010).

\subsection{Metodologia experimental}

Para execução dos experimentos foram utilizados os óleos vegetais de soja, girassol (lote: 1112), canola (lote: 0811), milho (lote: 1012), neem ${ }^{\circledR}$ (lote: 12029 e 13034) e mamona e os óleos minerais assist ${ }^{\circledR}$ $(\operatorname{cod} 3513)$ e naturol ${ }^{\circledR}$ (lote: 0446) os quais foram adquiridos em lojas do seguimento (Tabela 1), com exceção do óleo de mamona, o qual foi obtido a partir da prensagem a frio de sementes de $R$. communis da variedade IAC 80 e armazenada em recipiente recoberto com papel alumínio e hermeticamente fechado.

Tabela 1. Produtos comerciais utilizados nos bioensaios.

\begin{tabular}{|c|c|c|c|}
\hline $\begin{array}{c}\text { Norse } \\
\text { Comercial }\end{array}$ & Ingrediente ativo & Gropo quimico & Classe \\
\hline Neem ${ }^{6}$ & Nim & Botánico & Inseticida Nahinal \\
\hline Ausist ${ }^{2}$ & $\begin{array}{c}\text { Mistuia } \\
\text { Hidtocarbonetos }\end{array}$ & $\begin{array}{c}\text { Hidioncarbouetos } \\
\text { alifiticos }\end{array}$ & $\begin{array}{l}\text { Inseticiala } \\
\text { /Acaricida }\end{array}$ \\
\hline Naturote & $\begin{array}{l}\text { Mesturs } \\
\text { Hidfocarboestos }\end{array}$ & $\begin{array}{c}\text { Hidrocarbouetos } \\
\text { alifititicos }\end{array}$ & 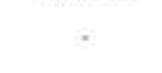 \\
\hline
\end{tabular}

Por ocasião dos bioensaios, a metodologia empregada foi baseada em Santa-Cecília et al. (2010), a qual utiliza discos foliares de $4,5 \mathrm{~cm}$ de diâmetro provenientes de folhas de café-conilon. Anteriormente, os discos foliares foram lavados em água clorada (5\%) e enxaguados em água destilada, secos e dispostos em uma lâmina de $5 \mathrm{~mm}$ ágar-água à $3 \%$ em placas de Petri ( $9 \mathrm{~cm}$ de diâmetro) com a face abaxial para cima. As placas foram fechadas com filme plástico PVC.

\subsection{Atividade inseticida dos óleos a P. citri}

Para a realização deste bioensaio foram preparadas as soluções na concentração de 3\% v/v dos óleos vegetais: Canola, Girassol, Soja, Milho, Neem ${ }^{\circledR}$ e Mamona; e dos óleos minerais: Naturol ${ }^{\circledR}$ e Assist $^{\circledR}$, contendo água destilada, Tween ${ }^{\circledR} 80(0,05 \%$ $\mathrm{v} / \mathrm{v})$ e acetona $(1 \% \mathrm{v} / \mathrm{v})$. A testemunha consistiu de água deionizada, Tween ${ }^{\circledR} 80$ $(0,05 \% \mathrm{v} / \mathrm{v})$ e acetona $(1 \% \mathrm{v} / \mathrm{v})$, completando um total de nove tratamentos, com 10 repetições por tratamento.

Cada arena constituiu uma repetição, na qual foram inoculadas 10 ninfas de 
segundo ínstar de $P$. citri, utilizando um pincel com cerdas macias. Dessa forma, foram utilizados 100 insetos/tratamento, perfazendo 900 insetos no bioensaio. Por ocasião da aplicação, os folíolos contidos nas arenas previamente montadas e infestadas com as ninfas foram pulverizadas na superfície abaxial com os respectivos óleos, utilizandose uma torre de Potter com pressão de 15 $\mathrm{lb} / \mathrm{pol}^{2}$ sendo pulverizado $6 \mathrm{~mL}$ da solução/repetição. Posteriormente, avaliou-se a mortalidade das ninfas diariamente por um período de três dias, constatando-se a morte com auxílio de um pincel de cerdas macias.

$\mathrm{O}$ experimento foi disposto em delineamento inteiramente casualizado (DIC). Os dados foram corrigidos pela fórmula de Abbott (1925), transformados por arcoseno da $\sqrt{ }(\mathrm{x} / 100)$ e submetidos à análise de variância, sendo as médias comparadas pelo teste de Scott-Knott, ao nível de 5\% de probabilidade.

\subsection{Estimativa da concentração letal $\left(C L_{50} e\right.$ CL90)}

Para estimar a concentração letal dos óleos foram considerados apenas os tratamentos com mortalidade acima de $90 \%$, com base no resultado do primeiro bioensaio. Posteriormente, para cada tratamento selecionado, foram avaliadas 10 concentrações espaçadas em escala logarítmica, cada qual com cinco repetições. Assim a CL de cada óleo foi composta por 500 insetos.

A condução experimental foi à mesma descrita no primeiro biensaio. As concentrações letais ( $\mathrm{CL}_{50}$ e $\mathrm{CL}_{90}$ ) foram estimadas, a partir dos dados de mortalidade, através da análise de Probit, usando o programa POLO-PC, determinando a CL com intervalo de confiança de 95\% (LEORA SOFTWORE, 1987).

\section{RESUltados E DiscuSSÃo}

\subsection{Soil chemical attributes}

Os resultados do bioensaio da atividade inseticida demonstram que óleo vegetal de Neem ${ }^{\circledR}$ e mineral Assist ${ }^{\circledR}$ obtiveram $100 \%$ de mortalidade de P. citri, seguido pelo óleo mineral Naturol ${ }^{\circledR}$ com $91,15 \%$ (Tabela 2). Os demais valores obtidos com a aplicação dos óleos vegetais variaram de 46,66\% para mamona a $81,63 \%$ para o girassol. Dentre esses, a mortalidade dos óleos vegetais de girassol e soja foram semelhantes, bem como o de milho e canola, já o óleo de mamona foi o que apresentou menor mortalidade e diferiu dos demais.

Tabela 2. Mortalidade corrigida de ninfas do segundo ínstar de Planococcus citri ocasionada por aplicação de óleos minerais e vegetais na concentração de 3\%. Temp.: $25 \pm$ $1{ }^{\circ} \mathrm{C}$, UR $70 \pm 10 \%$ e $12 \mathrm{~h}$ de fotofase.

\begin{tabular}{cc}
\hline Oleos Minerais e Vegetais & Mortalidade Corrigida $(\%)^{1,2}$ \\
\hline Neem $^{*}$ & $100,0 \pm 0,00 \mathrm{a}$ \\
Assist $^{*}$ & $100,0 \pm 0,00 \mathrm{a}$ \\
Naturol $^{*}$ & $91,1 \pm 2,84 \mathrm{~b}$ \\
Girassol & $81,6 \pm 3,22 \mathrm{c}$ \\
Soja & $75,8 \pm 2,97 \mathrm{c}$ \\
Milho & $66,2 \pm 3,59 \mathrm{~d}$ \\
Canola & $64,9 \pm 4,86 \mathrm{~d}$ \\
Mamona & $46,6 \pm 3,84 \mathrm{e}$ \\
\hline Estatistica & F $, 72=52,62 ; \mathrm{P}<0,0001 ; \mathrm{CV} \%=11,20$
\end{tabular}

${ }^{1}$ Médias ( \pm EP) seguidas pela mesma letra, na coluna, não diferem estatisticamente entre si pelo teste de Scott-Knott, a 5\% de probabilidade. ${ }^{2}$ Dados transformados em arcoseno da $\sqrt{ }(\mathrm{x} / 100)$.

Mediante os resultados encontrados no presente trabalho esse menor valor encontrado no tratamento com óleo de mamona pode estar associado às características do próprio óleo como a maior viscosidade, dificultando espalhar-se sobre a superfície de contato em relação aos demais (PAES et al., 2015). Assim a causa da mortalidade das ninfas de $P$. citri nesse bioensaio pode ser devido à ação do óleo por recobrir os espiráculos do inseto causando 
asfixia, como também apontam Lima et al. (2015) ser essa a causa da mortalidade de lagartas de Diaphania nitidalis tratadas com óleo de mamona. Além disso, para insetos de hábito picador-sugador o efeito residual sobre a superfície do substrato pode dificultar a locomoção e penetração do estilete para sucção de seiva.

No entanto para formulações mais modernas de óleos minerais derivados do petróleo, principalmente com a finalidade de minimizar a fitotoxidade em plantas, estudos apontam que a mortalidade dos insetos possa não estar baseada na asfixia (NAJARRODRÍGUEZ et al., 2008). Nestes casos os mecanismos pelo qual estes óleos saturados derivados do petróleo atuam parece causar perturbações celulares, tais como no sistema nervoso, toxidade celular e dessecação geral (NAJAR-RODRÍGUEZ et al., 2008).

Em avaliações de testes com pulgão e lagarta e aplicações de óleos minerais derivados do petróleo não foi constatado asfixia, pelo contrário houve uma possível interação destes óleos com a cutícula do inseto e deslocando-se através dela para dentro dos tecidos, havendo a partir disso uma alteração da permeabilidade da cutícula acarretando perda de água e dessecação (NAJARRODRÍGUEZ et al., 2008). Além disso, os autores constataram que ao penetrarem houve uma associação destes óleos com corpos gordurosos e o sistema nervoso central devido à natureza lipofílica do óleo, intervindo provavelmente na função sináptica e coordenação de atividades motoras (NAJARRODRÍGUEZ et al., 2008). Tais fatos possam explicar o destaque quanto a mortalidade encontrada neste trabalho para os tratamentos Assist $^{\circledR}$ e Naturol ${ }^{\circledR}$, por serem esses também produtos derivados de petróleo a base de hidrocarbonetos.

Contudo, a mortalidade também pode estar relacionada aos componentes químicos provenientes dos óleos vegetais (RAMOSLÓPEZ et al., 2010; ARNOSTI et al., 2011a; 2011b). A azadiractina, o principal princípio ativo do óleo de neem, geralmente causa inibição do crescimento e alteram a metamorfose de larvas de lepidoptera, coleoptera, hymenoptera e diptera e ninfas de orthoptera, bem como atua como antialimentar, principalmente em adultos de coleoptera, hemiptera e orthoptera (AGUIARMENEZES, 2005). O óleo de mamona é constituído por ácidos graxos como palmítico $(1,2 \%)$, esteárico $(0,7 \%)$, oleico $(3,2 \%)$, linoleico $(3,4 \%)$, linolênico $(0,2 \%)$ e o ricinoleico $(89,4 \%)$, sendo provável que também esses compostos possam estar envolvidos com a mortalidade (JENA e GUPTA, 2012; CELESTINO et al., 2015).

Segundo o Ministério da Agricultura, Pecuária e Abastecimento (MAPA) para $P$. citri existem alguns óleos minerais registrados, são eles: Assist ${ }^{\circledR}$ na cultura dos citros; Triona ${ }^{\circledR}$ na cultura da uva e Iharol $^{\circledR}$ para cacau, entretanto para a cultura do café ainda não há nenhum registro tanto de óleos quanto de inseticidas (MAPA, 2020).

Em busca de estratégias para o manejo desta praga no cafeeiro, Santa-Cecília et al. (2010) avaliaram em laboratório 232 extratos com base em 186 diferentes espécies de plantas com atividade inseticida, no entanto apenas o do abacateiro, Persea americana Mill. (Lauraceae), foi selecionado por causar a maior mortalidade. Isso reforça a resposta encontrada no presente trabalho com os óleos vegetais e minerais, cujo a grande parte apresentou mortalidade acima de 70\%, apresentando perspectivas promissoras para manejo.

O potencial inseticida de neem também foi testado para outras pragas do cafeeiro, como o bicho-mineiro Leucoptera coffeella Guérin-Menéville \& Perrottet (Lyonetiidae), ácaro-vermelho-do-cafeeiro Oligonychus ilicis Mc Gregor (Tetranychidae), a broca-do-café Hypothenemus hampei Ferrari (Scolytidae), apresentando potencial de controle, e ainda sendo seletivo ao fungo Beauveria bassiana Balsamo Vuillemim (Cordycipitaceae) e ao ácaro predador Iphiseiodes zuluagai Denmark \& Muma (Phytoseiidae) (VENZON et al., 
2005; ZORZETTI et al., 2012). Tal fato representa uma vantagem em programas de Manejo Integrado de Pragas onde um mesmo método de controle pode apresentar eficiência para um conjunto de insetos-praga pertinentes à cultura, além de apresentar seletividade aos inimigos naturais, haja visto que o óleo de neem obteve excelente resultado para $P$. citri nessa pesquisa, uma das principais pragas do cafeeiro.

Estudos sobre fatores que afetam a mortalidade natural de $P$. citri verificaram que o terceiro ínstar foi o estádio crítico de mortalidade e o fator chave que mais contribuiu para isso foi a predação, caso esse fator fosse desconsiderado da mortalidade total a população de $P$. citri aumentaria em $459 \%$ (SILVA, 2011). Entretanto, devido ao uso indiscriminado de inseticidas utilizados no controle de Planococcus sp., como para outras pragas e doenças que ocorrem simultaneamente, podem afetar severamente os predadores e parasitoides. Desta forma optar por produtos seletivos aos inimigos naturais, tais como óleos minerais, despertam interesse para a pesquisa (GRAVENA, 2003).

Esse interesse fica evidente nos estudos com óleo de girassol, canola, milho, soja e Agrex $^{\circledR}$ para o controle de Bemisia tabaci Gennadius biótipo B (Aleyrodidae) e Thrips tabaci Lindeman (Thripidae); óleos de mamona, neem e soja obtendo um controle da mosca-branca-do-cajueiro, Aleurodicus cocois Curtis (Aleyrodidae), acima de $91 \%$ sem afetar as abelhas polinizadoras (SILVA et al., 2007; COSTA et al., 2010). No cultivo de palma-gigante a eficiência do óleo de laranja para o controle da cochonilha-do-carmim, Dactylopius opuntiae Cockerell (Dactylopiidae), foi em torno de $90 \%$ e ainda constatou seletividade do produto para adultos das joaninhas Cycloneda sanguínea Linnaeus (Coccineliidae) e Scymnus intrusus Horn (Coccineliidae) (LOPES et al., 2009).

Os valores de $\mathrm{CL}_{50}$ obtidos com os óleos foram de $0,67 \%$ (v/v) para o $\mathrm{Neem}^{\circledR}$, $0,89 \%$ para o Assist $^{\circledR}$ e de $0,76 \%$ para Naturol $^{\circledR}$ (Tabela 3). Estes valores não diferiram estatisticamente de acordo com o intervalo de confiança da CL50 a $95 \%$ de probabilidade (IC a 95\%). Desta forma, outros testes devem ser realizados para a escolha do mais adequado, como por exemplo, seletividade a inimigos naturais, atuação do óleo a campo exposto às condições ambientais e viabilidade econômica do produto.

Tabela 3. Toxicidade de óleos minerais e vegetal sobre ninfas do segundo ínstar de Planococcus citri. Temp.: $25 \pm 1^{\circ} \mathrm{C}$, UR $70 \pm$ $10 \%$ e $12 \mathrm{~h}$ de fotofase.

\begin{tabular}{|c|c|c|c|c|c|c|}
\hline Óleos & $N^{\prime}$ & $\begin{array}{l}\text { Inclinacis } \\
\pm E P^{2}\end{array}$ & $\begin{array}{c}\text { CL ud ac a 9596r } \\
\text { (7o viv) }\end{array}$ & 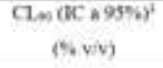 & $\mathrm{GL}^{\prime}$ & $x^{2+1}$ \\
\hline Nivemn & 375 & $3,09=0,49$ & $\begin{array}{c}0.67 \\
(0.46-0,83)\end{array}$ & $\begin{array}{c}1.74 \\
(1,36-2.84)\end{array}$ & 5 & 5,21 \\
\hline Aveste ${ }^{*}$ & 421 & $2,46 \pm 0,48$ & $\begin{array}{c}0.89 \\
(0,50-1,19)\end{array}$ & $\begin{array}{c}2,82 \\
(2,03-6,13)\end{array}$ & 6 & 6.93 \\
\hline Natusol" & 427 & $1, \pi 0 \pm 0,17$ & $\begin{array}{c}0,76 \\
(0,60-0,96)\end{array}$ & $\begin{array}{c}4,35 \\
(3,16-6,75)\end{array}$ & 6 & 3,76 \\
\hline
\end{tabular}

${ }^{1}$ Número de insetos usados no teste.

${ }^{2}$ Erro-padrão.

${ }^{3}$ Intervalo de confiança das $\mathrm{CL}_{50}$ e $\mathrm{CL}_{90}$ a $95 \%$ de probabilidade.

${ }^{4}$ Número de graus de liberdade.

${ }^{5}$ Teste qui-quadrado

Para H. hampei, outra praga do cafeeiro, a $\mathrm{CL}_{50}$ encontrada para o óleo de $\mathrm{Neem}^{\circledR}$ foi de $6,71 \%$ (CELESTINO et al., 2016), essas diferenças nos valores de concentração letal possivelmente estão relacionadas à rusticidade externa do exoesqueleto e a vulnerabilidade à ação do óleo devido as características desses insetos, tratando-se de um coleóptero e outro da ordem hemíptera.

Em relação à CL 90 o óleo de mineral Assist ${ }^{\circledR}$ não diferiu estatisticamente dos óleos de Neem ${ }^{\circledR}$ e Naturol ${ }^{\circledR}$ de acordo com o IC a $95 \%$. Já o Naturol ${ }^{\circledR}$ obteve mais que o dobro do valor de $\mathrm{CL}_{90}$ encontrado com o óleo de $\mathrm{Neem}^{\circledR}, 4,35 \%$ e $1,74 \%$ respectivamente, diferindo estatisticamente entre si. Além disso, a curva de concentração versus 
mortalidade do óleo de $\mathrm{Neem}^{\circledR}$ foi a que apresentou maior inclinação, isto demonstra que pequenas variações na concentração do óleo provocam maiores variações na mortalidade de $P$. citri, apresentado uma resposta mais homogenia da população a esse óleo.

É válido ressaltar que antes de optar pelo uso dos óleos, também devem ser avaliadas reações de fitotoxicidade da planta, procurando informações quanto as dosagens compatíveis aos respectivos estádios fenológicos, além das características e qualidade do óleo (RODRIGUES e CHILDERS, 2002; ANDRADE et al., 2010; ATAIDE et al., 2018). Estudos à campo devem ser implementados para validar a eficiência dos óleos e adequá-los para programas de Manejo Integrado de Pragas, minimizando assim os impactos gerados com o uso indiscriminado de produtos químicos sintéticos.

\section{CONCLUSÃo}

Os óleos minerais Assist ${ }^{\circledR}$ e Naturol ${ }^{\circledR} \mathrm{e}$ os óleos vegetais $\mathrm{Neem}^{\circledR}$, girassol, soja, milho, canola e mamona avaliados na concentração de 3\% apresentam atividade inseticida a $P$. citri, sendo que os óleos Assist ${ }^{\circledR}$ e Neem ${ }^{\circledR}$ ocasionaram $100 \%$ de mortalidade. Os valores de $\mathrm{CL}_{50}$ obtidos com os óleos de $\mathrm{Neem}^{\circledR}, \quad$ Assist $^{\circledR}$ e Naturol $^{\circledR}$ foram semelhantes.

\section{Agradecimentos}

À Coordenação de Aperfeiçoamento de Pessoal de Nível Superior (Capes), à Fundação de Amparo à Pesquisa e Inovação do Espírito Santo (FAPES) e ao Conselho Nacional de Desenvolvimento Científico e Tecnológico (CNPq) pelo apoio financeiro para o desenvolvimento da pesquisa.

\section{REFERÊNCIAS}

ABBOTT, W. S. A method of computing the effectiveness of an insecticide. Journal of Economic Entomology, v. 18, n. 2, p. 265266, 1925.

AGUIAR-MENEZES, E. de L. A. Inseticidas Botânicos: Seus princípios ativos modo de ação e uso agrícola. Embrapa Agrobiologia, Seropédica, 2005. (Embrapa Agrobiologia. Documentos, 205).

ANDRADE, D. J. de.; FERREIRA, M. da C.; SANTOS, N. C. dos. Efeito da adição de óleos ao acaricida cyhexatin sobre ácaro Brevipalpus phoenicis e na retenção de calda por folhas de citros. Revista Brasileira de Fruticultura, v. 32, n. 4, p. 1055-1063, 2010.

ARNOSTI, A.; BRIENZA, P. D.; FURQUIM, K. C. S.; CHIERICE, G. O.; NETO, S. C.; BECHARA, G. H.; SAMPIERI, B. R.; CAMARGO-MATHIAS, M. I. Effects of Ricinus communis oil esters on salivary glands of Rhipicephalus sanguineus (Latreille, 1806) (Acari: $\quad$ Ixodidae). Experimental Parasitology, v. 127, n. 2, p. 569-574, 2011 a.

ARNOSTI, A.; BRIENZA, P. D.; FURQUIM, K. C. S.; CHIERICE, G. O.; BECHARA, G. H.; CALLIGARIS, I. M.; CAMARGOMATHIAS, M. I. Effects of ricinoleic acid esters from castor oil of Ricinus communis on the vitellogenesis of Rhipicephalus sanguineus (Latreille, 1806) (Acari: Ixodidae) ticks. Experimental Parasitology, v. 127, n. 2, p. 575-580, 2011 b.

ATAIDE, J.O.; HUVER, A.; DEOLINDO, F.D.; HOLTZ, F.G.; ZAGO, H.B.; MENINI, L. Avaliar a atividade acaricida dos óleos essenciais de Zingiber officinale e Rosmarinus officinalis por fumigação sobre fêmeas de Tetranychus urticae. Acta Ambiental Catarinense, v. 15, n. 1/2, p. 57-65, 2018. 
BESTETE, L. R.; PRATISSOLI, D.; QUEIROZ, V. T. de; CELESTINO, F. N.; MACHADO, L. C. Toxicidade de óleo de mamona a Helicoverpa zea e a Trichogramma pretiosum. Pesquisa Agropecuária Brasileira, v. 4, n. 8, p. 791-797, 2011.

CELESTINO, F. N.; PRATISSOLI, D.; MACHADO, L. M.; COSTA, A. V.; SANTOS JUNIOR, H. J. G. DOS; ZINGER, F. D. Toxicidade do óleo de mamona à brocado-café [Hypothenemus hampei (Ferrari) (Coleoptera: Curculionidae: Scolytinae)]. Coffee Science, v. 10, n. 3, p. 329 - 336, 2015.

CELESTINO, F. N.; PRATISSOLI, D.; MACHADO, L. M.; SANTOS JUNIOR, H. J. G. DOS; QUEIROZ, V. T. de; MARDGAN, L. Control of coffee berry borer, Hypothenemus hampei (Ferrari) (Coleoptera: Curculionidae: Scolytinae) with botanical insecticides and mineral oils. Acta Scientiarum, v. 38, n. 1, p. 1 - 8, 2016.

COMPANHIA NACIONAL DE ABASTECIMENTO (CONAB). Acompanhamento da safra brasileira de café: safra 2020, primeiro levantamento, janeiro/2020. Brasília: CONAB, 2020.

COSTA, G. M.; BOIÇA JUNIOR, A. L.; JESUS, F. G. de; CHAGAS FILHO, N. R. Efeito do uso de óleos vegetais, associados ou não a inseticida, no controle de Bemisia tabaci (Gennadius, 1889) e Thrips tabaci (Lindeman, 1888), em fejoeiro, na época "das águas". Bioscience Journal, v. 26, n. 1, p. 15-23, 2010.

FITE, T.; TEFERA, T.; NEGERI, M,; DAMTE, T. Effect of Azadirachta indica and Milletia ferruginea extracts against Helicoverpa armigera (Hubner) (Lepidoptera: Noctuidae) infestation management in chickpea. Cogent Food \& Agriculture, v.6, p. 1 - 15, 2020.

FORNAZIER, M. J.; SANTA-CECÍLIA, L. V. C.; MARTINS D. dos S. Cochonilhasfarinhentas associadas ao café conillon. In: ZAMBOLIM, L. Tecnologias para produção do Café Conilon. Viçosa: UFV, 2009. p. 125-152.

GRAVENA, S. Manejo ecológico da cochonilha-branca dos citros, com ênfase no controle biológico pela joaninha Cryptolaemus montrouzieri. Laranja, v. 24, n. 1, p. 71-82, 2003.

ISMAN, M. B. Botanical insecticides, deterrents, and repellents in modern agriculture and an increasingly regulated world. Annual Review of Entomology, v.51, p. 45-66, 2006.

J

ENA, J.; GUPTA, A. K. Ricinus communis Linn: a phytopharmacological review. International Journal of Pharmacy and Pharmaceutical Sciences, v. 4, n. 4, p. 25-29, 2012.

KUMAR, R.; KRANTHI, S.; NAGRARE, V. S.; MONGA, D.; KRANTHI, K. R.; RAO, N.; SINGH, A. Insecticidal activity of botanical oils and other neem-based derivatives against whitefly, Bemisia tabaci (Gennadius) (Homoptera: Aleyrodidae) on cotton. International Journal of Tropical Insect Science, v.39, n.3, p. 203 - 210, 2019.

LEORA SOFTWARE. POLO-PC: An user's guide to probit or logit analysis. Berkeley, CA, USA: LeOra Software, 1987. 22p.

LIMA, V. L. DE S.; CELESTINO, F. N.; PRATISSOLI, D. DALVI, L. P.; CARVALHO, J. R. DE; PAES, J. P. P. Atividade inseticida do óleo de mamona sobre Diaphania nitidalis (Stoll) (Lepidoptera: 
Pyralidae). Revista Brasileira de Ciências Agrárias, v.10, n.3, p.347-351, 2015.

LOPES, E. B.; BRITO, C. H. de; ALBUQUERQUE, I. C. de; BATISTA, J. de L. Desempenho do óleos de laranja no controle da cochonilha-do-carmim em palma gigante. Engenharia Ambiental, v. 6, n. 1, p. 252-258, 2009.

\section{MINISTÉRIO DA AGRICULTURA, PECUÁRIA E ABASTECIMENTO} (MAPA). AGROFIT- Sistema de Agrotóxico Fitossanitários. Disponível em: http://agrofit.agricultura.gov.br/agrofit_cons/ principal_agrofit_cons. Acesso em: 31 jan. 2020.

MRUTHUNJAYASWAMY, P.; THIRUVENGADAM, V.; KUMAR, J. S. Detection of insecticide resistance in field populations of citrus mealybug Planococcus citri (Risso) (Hemiptera: Pseudococcidae). Indian Journal of Experimental Biology, v.57, p. 453-442, 2019.

NAJAR-RODRÍGUEZ, A. J.; LAVIDIS, N. A.; MENSAH, R. K.; WALTER, G. H. The toxicological effects of petroleum spray oils on insects - Evidence for an alternative mode of action and possible new control options. Food and Chemical Toxicology, v.46, p. 3003-3014, 2008.

NISHIJIMA, M.; SAES, M. S. M.; POSTALI, F. A. S. Análise de concorrência no mercado mundial de café verde. RESR, v. 50, n. 1, p. 069-082, 2012.

PAES, J. B.; SOUZA, A. D. DE; LIMA, C. R. DE; SANTANA, G. M. Rendimento e Características Físicas dos Óleos de Nim (Azadirachta indica) e Mamona (Ricinus communis). Floresta e Ambiente, v. 22, n. 1, p. $134-139,2015$.
PICANÇO, M.C; SANTANA Jr., P. A.; SILVA, G. A.; LOPRES, M.C.; ARAÚJO, T.A.; SILVA, G.A.R. Manejo Integrado de Pragas. In: SAKIYAMA, N.S.; MARTINEZ, H.E.P.; TOMAZ, M.A.; BORÉM, A. Café arábica do Plantio à Colheita. Viçosa, MG: Ed. UFV, 2015. p. 151-173.

PIMENTEL, L. C. F.; CHAVES, C. R.; FREIRE, L. A. A.; AFONSO, J. C. O inacreditável emprego de produtos químicos perigosos no passado. Química nova, v. 29, n. 5, p. 1138-1149, 2006.

RAMOS-LÓPES, M. A.; PÉREZ, S. G.; GUEVARA-FEFER, P.; ZAVALASÁNCHEZ, M. A. Activity of Ricinus communis (Euphorbiaceae) against Spodoptera frugiperda (Lepidoptera: Noctuidae). African Journal of Biotechnology, v. 9, n. 9, p. 1359-1365, 2010. RODRIGUES, J. C. V.; CHILDERS, C. C. Óleos no manejo de pragas e doenças em Citros. Laranja, v. 32, n. 1, p. 77-100, 2002.

RONDELLI, V. M.; PRATISSOLI, D.; POLANCZYK, R. A.; MARQUES, E. J.; STURM, G. M.; TIBURCIO, M. O. Associação do óleo de mamona com Beauveria bassiana no controle da traça-dascrucíferas. Pesquisa Agropecuária Brasileira, v. 46, n. 2, p. 212-214, 2011.

SANCHES, N. F.; CARVALHO, R. da S. Procedimentos para manejo da criação e multiplicação do predador exótico Cryptolaemus montrouzieri. Embrapa Mandioca e Fruticultura, p. 1-5, 2010. (Circular Técnica, 99).

SANTA-CECÍLIA, L. V. C.; REIS, P. R.; SOUZA, J. C. Sobre a nomenclatura das espécies de cochonilhas-farinhentas do cafeeiro nos Estados de Minas Gerais e Espírito Santo. Neotropical Entomology, v. 31, n. 2, p. 333-334, 2002. 
SANTA-CECÍLIA, L. V. C.; SANTACECÍlLIA, F. V.; PEDROSO, E. do C.; SOUSA, M. V. de. ABREU, F. A.; OLIVEIRA, D. F.; CARVALHO, G. A. Extratos de plantas no controle de Planococcus citri (Risso, 1813) (Hemiptera: Pseudococcidae) em cafeeiro. Coffee Science, v. 5, n. 3, p. 283-293, 2010.

SILVA, N. R. da. Fatores de mortalidade natural da cochonilha-farinhenta Planococcus citri no cafeeiro. 2011. 29 p. Dissertação (Mestrado em Fitotecnia)-UFV. Viçosa, 2011.

SILVA, P. H. S. da; CARNEIRO, J. da S. CASTRO, M. de J. P de; LOPES, M. T. do R. Ação biocida de óleos vegetais em ovos e ninfas da mosca-branca-do-cajueiro e operárias de Apis mellifera L. Embrapa Meio-Norte, 2007 (Comunicado Técnico 205).

TIILIKKALA, K.; LINDQVIST, I.; HAGNER, M.; SETÄLÄ, H.; PERDIKIS, D. Use of botanical pesticides in modern plant protection. Pesticides in the Modern World - Pesticides Use and Management, p. 259272, 2011.

VENZON, M.; ROSADO, M. C.; FADINI, M. A.M.; CIOCIOLA Jr. A. I.; PALLINI, A. The potential of Neem Azal for the control of coffee leaf pests. Crop Protection, v. 24, p. 213-219, 2005.

ZIBAEE, A. Botanical insecticides and their effects on insect biochemistry and immunity. Pesticides in the Modern World - Pests Control and Pesticides Exposure and Toxicity Assessment, p. 55-68, 2011.

ZORZETTI, J.; NEVES, P. M. O. J.; CONSTANSKI, K. C.; SANTORO, P. H.; FONSECA, I. C. B. Plant extracts on
Hypothenemus hampei (Coleptera:

Curculionidae) and Beauveria bassiana. Semina: Ciências Agrárias, v. 33, n. 1, p. 2849-2862, 2012.

Submetido: 05/03/2020.

Aceito: 26/03/2020. 